\title{
Electron spin resonance observation of charge carrier concentration in organic field-effect transistors during device operation
}

\author{
Hisaaki Tanaka, ${ }^{1}$ Masataka Hirate, ${ }^{1}$ Shun-ichiro Watanabe, ${ }^{1}$ Kazuaki Kaneko, ${ }^{1}$ Kazuhiro Marumoto, ${ }^{2,3}$ Taishi Takenobu, ${ }^{3,4}$ \\ Yoshihiro Iwasa, ${ }^{5}$ and Shin-ichi Kuroda ${ }^{1}$ \\ ${ }^{1}$ Department of Applied Physics, Nagoya University, Chikusa, Nagoya 464-8603, Japan \\ ${ }^{2}$ Institute of Materials Science, University of Tsukuba, Tsukuba, Ibaraki 305-8573, Japan \\ ${ }^{3}$ Japan Science and Technology Agency (JST), PRESTO, Kawaguchi, Saitama 322-0012, Japan \\ ${ }^{4}$ Department of Applied Physics, Waseda University, 3-4-1, Okubo, Shinjuku, Tokyo 169-8555, Japan \\ ${ }^{5}$ Quantum Phase Electronics Center and Department of Applied Physics, University of Tokyo, Tokyo 113-8656, Japan
}

(Received 21 September 2012; published 18 January 2013)

\begin{abstract}
Charge carrier concentration in operating organic field-effect transistors (OFETs) reflects the electric potential within the channel, acting as a key quantity to clarify the operation mechanism of the device. Here, we demonstrate a direct determination of charge carrier concentration in the operating devices of pentacene and poly(3-hexylthiophene) (P3HT) by field-induced electron spin resonance (FI-ESR) spectroscopy. This method sensitively detects polarons induced by applying gate voltage, giving a clear FI-ESR signal around $g=2.003$ in both devices. Upon applying drain-source voltage, carrier concentration decreases monotonically in the FET linear region, reaching about $70 \%$ of the initial value at the pinch-off point, and stayed constant in the saturation region. The observed results are reproduced well from the theoretical potential profile based on the gradual channel model. In particular, the carrier concentration at the pinch-off point is calculated to be $\beta /(\beta+1)$ of the initial value, where $\beta$ is the power exponent in the gate voltage $\left(V_{\mathrm{gs}}\right)$ dependence of the mobility $(\mu)$, expressed as $\mu \propto V_{\mathrm{gs}}^{\beta-2}$, providing detailed information of charge transport. The present devices show $\beta=2.6$ for the pentacene and $\beta=2.3$ for the $\mathrm{P} 3 \mathrm{HT}$ cases, consistent with those determined by transfer characteristics. The gate voltage dependence of the mobility, originating from the charge trapping at the device interface, is confirmed microscopically by the motional narrowing of the FI-ESR spectra.
\end{abstract}

DOI: 10.1103/PhysRevB.87.045309

PACS number(s): 73.61.Ph, 73.50.Gr, 76.30.-v, 85.30.Tv

\section{INTRODUCTION}

Organic field-effect transistors (OFETs) using $\pi$ conjugated molecules and polymers are attracting considerable attention owing to their potential applications for low-cost, flexible, and large area electronic circuits. ${ }^{1-4}$ Performance of OFETs has been improved remarkably, showing the mobility higher than $1 \mathrm{~cm}^{2} / \mathrm{Vs}$, even in polycrystalline thin films. This can be ascribed to the highly sophisticated design of the semiconductor materials as well as the development of fabrication techniques such as chemical treatment of the device interface by using self-assembled monolayers (SAMs). ${ }^{5-15}$ On the other hand, understanding of the basic physical processes, such as charge injection or transport, as well as the electronic state of charge carriers at the semiconductor/insulator interface is still under progress. Thus the microscopic observation of charge carriers during the device operation is an essential subject in understanding the operation principle of the device, which may also contribute to design highly efficient devices.

In principle, device operation of OFETs is understood based on the conventional model using gradual channel approximation (GCA). ${ }^{4,16,17}$ According to the model, a potential distribution is induced within the channel by applying gatesource and source-drain voltages, $V_{\mathrm{gs}}$ and $V_{\mathrm{ds}}$, respectively, dominating the FET output current. The shape of the potential profile has been experimentally observed in the operating OFET by using scanning Kelvin probe microscopy (SKPM), giving linear and superlinear distributions corresponding to the linear and the saturation regions of FET output characteristics, respectively. ${ }^{18}$ The potential profile also gives detailed information of charge transport, such as contact resistance, ${ }^{18-21}$ charge trapping at grain boundaries,${ }^{20-22}$ as well as charge concentration dependence of the mobility. ${ }^{23-25}$ However, SKPM provides no spectroscopic information of charge carriers. Since the charge distribution within the channel is proportional to the potential distribution within the framework of GCA, determination of charge carrier concentration by the spectroscopic method is a promising alternative approach to finding the operation principle of the device together with the microscopic information of charge carriers.

Field-induced electron spin resonance (FI-ESR) spectroscopy, developed by our group, is a particularly suitable method for this purpose owing to its high sensitivity $\left(<10^{11}\right.$ spins) to detect the spin of accumulated carriers at the insulator interface. ${ }^{26}$ So far, this method has been applied to various OFETs of poly(3-alkylthiophene), ${ }^{26-30}$ pentacene, ${ }^{31-33}$ thienothiophene-based high-mobility materials, ${ }^{34,35}$ and rubrene single crystals, ${ }^{36}$ etc., giving microscopic information such as spin-charge relation, carrier wave function, motional effect of charge carriers, and local molecular orientation at the device interface. In particular, direct determination of spin concentration within the channel during device operation played a crucial role in clarifying the operation mechanism of OFETs, as was demonstrated for P3HT transistors using top-contact geometry, ${ }^{37}$ and subsequently, for rubrene single-crystal transistors with bottom-contact geometry; ${ }^{38}$ the spin concentration exhibited a clear decrease by applying $V_{\mathrm{ds}}$ together with $V_{\mathrm{gs}}$ in the linear region, whereas no $V_{\mathrm{ds}}$ dependence was observed 
for the saturation region. This result agreed qualitatively with that expected from the change of potential profiles. However, in the previous study of the P3HT transistor, the FI-ESR signal of charge carriers accumulated underneath the top electrodes overlapped with that of channel carriers, resulting in slight underestimation of the modulation effect of channel carriers by the application of drain voltage, as discussed in this paper.

In this study, we applied the FI-ESR technique to the operating OFET of pentacene fabricated on the $\mathrm{SiO}_{2}$ insulator, which has better surface flatness than $\mathrm{Al}_{2} \mathrm{O}_{3}$ or parylene insulators adopted in the earlier FI-ESR studies of pentacene. ${ }^{31,32}$ In order to obtain the accurate spin concentration within the channel, we fabricated the top-contact FETs with the length of the top electrodes to be sufficiently small compared to the channel length. Furthermore, the spin concentration underneath the electrodes was subtracted from the total concentration. Thus obtained spin concentration of channel carriers (polarons) exhibited a clear $V_{\mathrm{ds}}$ dependence in the linear region, reaching $\sim 70 \%$ of the initial value at the pinch-off point, whereas no $V_{\mathrm{ds}}$ dependence was observed in the saturation region. The result was reproduced well by the theoretical potential profile considering the power-law dependence of the mobility on the charge concentration. The concentration-dependent mobility was further confirmed from the transfer characteristics of the same device. Similar results were also obtained for P3HT transistors. Moreover, the observed FI-ESR line width exhibited an enhanced motional narrowing for higher spin concentrations, as in the case of pentacene transistors fabricated on parylene insulators, ${ }^{32,33}$ providing microscopic evidence for faster carrier motion for higher charge concentration due to the charge trapping at the interface.

The organization of the paper is as follows. The device fabrication methods and experimental details are described in Sec. II. We first present the basic FI-ESR results of pentacene transistors obtained without applying $V_{\mathrm{ds}}$ in Sec. III A. In this section, gate voltage dependence of the spin concentration of pentacene $\pi$-electron carriers as well as the motionally narrowed ESR line width are presented. In addition, local molecular orientation of pentacene at the insulator interface is confirmed by the angular dependence of the $g$ value. These basic characteristics of pentacene at the $\mathrm{SiO}_{2}$ interface are compared with those reported by earlier studies using $\mathrm{Al}_{2} \mathrm{O}_{3}$ or parylene insulators, which shows that the FET channels of the present devices are well organized and suitable for the analysis. The main results of $V_{\mathrm{ds}}$ dependence of the spin concentration are presented in Sec. III B, both for pentacene and P3HT devices. The results are compared with those expected from the theoretical potential profile considering gate voltage dependence of the mobility. The conclusions are given in Sec. IV.

\section{EXPERIMENTAL}

Pentacene powder was purchased from Aldrich Co. Ltd. and was used without further purification. Pentacene thin films with typical thickness of $100 \mathrm{~nm}$ were vapor-deposited on the $\mathrm{n}^{+}$-Si wafer (10-20 $\Omega \mathrm{cm},\langle 100\rangle$ axis, $0.2 \mathrm{~mm}$ thick) with (a)
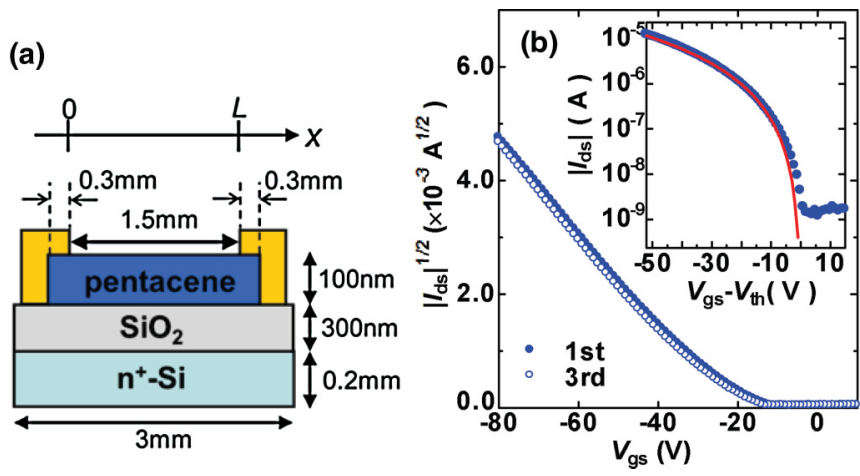

FIG. 1. (Color online) (a) Schematic illustration of the device structure and typical dimensions of the pentacene FET. The $x$ axis indicates the position within the channel from the source electrode edge. (b) Transfer characteristics of a pentacene FET obtained by the first (solid circles) and the third (open circles) scans. The drain voltage is $V_{\mathrm{ds}}=-50 \mathrm{~V}$. Inset shows the logarithmic plot of the drain current with respect to the effective gate voltage of $V_{\mathrm{gs}}-V_{\mathrm{th}}$, with a threshold voltage of $V_{\text {th }}=-20 \mathrm{~V}$. The solid curve represents the fitting by Eq. (4) with $\beta=2.6$ for the saturation regime.

a 300-nm-thick thermally grown $\mathrm{SiO}_{2}$ insulator treated with hexamethyldisilazane. Au electrodes $(30 \mathrm{~nm})$ were vapordeposited on the pentacene layer to form the top-contact geometry. Figure 1(a) shows the schematic illustration of the device. Typical dimensions of the device were channel length $(L)$ of $1.5 \mathrm{~mm}$ and channel width $(W)$ of $20 \mathrm{~mm}$. The length of the film underneath a top electrode was $0.3 \mathrm{~mm}$, much smaller than $L$. The P3HT transistors were prepared in the same way as the pentacene device except for the drop-cast process to form thin films. Field-effect transistor characteristics were measured by using a Keithley 2612A source measure unit. Figure 1(b) shows typical transfer characteristics of a pentacene FET. We obtain standard device operation with the mobility $(\mu)$ and threshold voltage $\left(V_{\mathrm{th}}\right)$ of $0.1 \mathrm{~cm}^{2} / \mathrm{Vs}$ and $-20 \mathrm{~V}$, respectively, as determined following the conventional procedures using GCA in the saturation regime. ${ }^{16}$ The maximum mobility was $\mu_{\max }=0.6 \mathrm{~cm}^{2} / \mathrm{Vs}$ at room temperature in this study. On the other hand, the mobility exhibits certain gate voltage dependence, as is discussed later. The operational stability of the device is satisfactory with a small shift of $V_{\text {th }}$ between the first and third scans, as shown in Fig. 1(b).

Electron spin resonance measurements were performed by using a Bruker E-500 spectrometer equipped with $\mathrm{TE}_{011}$ cylindrical cavity. The microwave frequency was directly monitored by the frequency counter at each scan. The magnetic field was recorded with the accuracy of $\pm 0.01 \mathrm{G}$ by using a nuclear magnetic resonance teslameter to determine the precise $g$ values. The FI-ESR signal of injected carriers was clearly obtained by subtracting the background ESR signal at $V_{\mathrm{gs}}=30 \mathrm{~V}$ from that obtained under the negative bias voltage. The spin concentration was determined within the error of $10 \%$ for the high $V_{\mathrm{gs}}$ region from the twiceintegration of the first-derivative ESR signal calibrated by that of $\mathrm{CuSO}_{4} \cdot 5 \mathrm{H}_{2} \mathrm{O}$. 

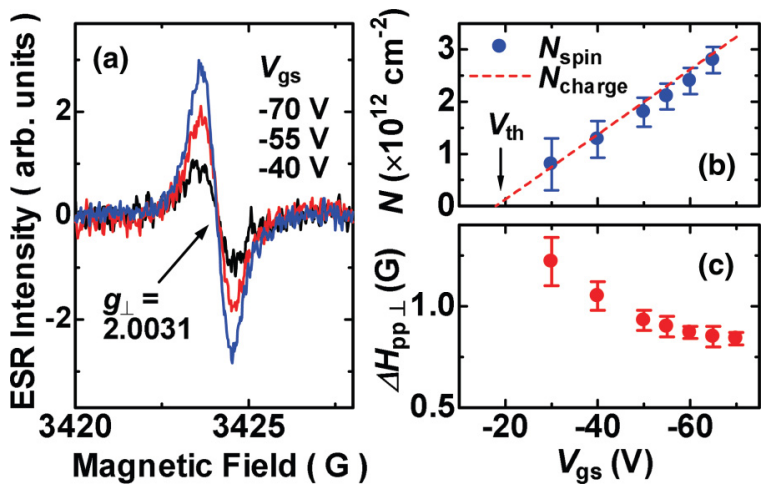

FIG. 2. (Color online) Gate voltage dependence of (a) firstderivative FI-ESR spectra and (b) spin concentration $\left(N_{\text {spin }}\right.$; solid circles) together with the charge concentration ( $N_{\text {charge }}$; dashed line) and (c) peak-to-peak line width obtained for a pentacene FET without applying $V_{\mathrm{ds}}$. The magnetic field is applied perpendicular to the substrate.

\section{EXPERIMENTAL RESULTS AND DISCUSSIONS}

\section{A. FI-ESR measurements on metal-insulator-semiconductor configuration $\left(V_{\mathrm{ds}}=\mathbf{0}\right)$}

In this subsection, we show the basic FI-ESR characteristics of the pentacene FET fabricated on the $\mathrm{SiO}_{2}$ insulator. The results compare well with those reported by the previous studies using $\mathrm{Al}_{2} \mathrm{O}_{3}$ or parylene insulators, which shows that the present devices are suitable for the analysis carried out in Sec. III B.

Figure 2(a) shows the gate voltage dependence of the first-derivative FI-ESR spectra obtained with the source and drain electrodes short-circuited. In this case, the device acts as a metal-insulator-semiconductor (MIS) capacitor. The external magnetic field is perpendicular to the substrate. We observe clear FI-ESR signals of $\pi$-electron carriers of pentacene around $g=2.003$. Figure 2(b) shows the $V_{\mathrm{gs}}$ dependence of the accumulated spin concentration $\left(N_{\text {spin }}\right)$ at the interface. Here, $N_{\text {spin }}$ increases linearly with a finite threshold voltage of $V_{\mathrm{th}}=-20 \mathrm{~V}$, consistent with that determined by the transfer characteristics in Fig. 1(b). The obtained spin concentration agrees well with the charge concentration ( $\left.N_{\text {charge }}\right)$, as shown by the dashed line in Fig. 2(b). Here, $N_{\text {charge }}$ is calculated from the relation $e N_{\text {charge }}=C_{i}\left|V_{\mathrm{gs}}-V_{\mathrm{th}}\right|$, where $e$ is the elementary charge and $C_{i}$ is the capacitance of the insulator $\left(C_{i}=11.3 \mathrm{nF} / \mathrm{cm}^{2}\right)$. The agreement between $N_{\text {spin }}$ and $N_{\text {charge }}$ indicates that all the injected charge carries have spin $1 / 2$, that is, polarons. This result is independent of the insulator material. ${ }^{31,32}$

As the spin concentration increases, peak-to-peak ESR line width $\left(\Delta H_{\mathrm{pp}}\right)$ exhibits clear narrowing, as shown in Fig. 2(c). Similar behavior has been reported by Matsui et al. in the pentacene FET with parylene insulator. ${ }^{31}$ They ascribed this behavior to the motional narrowing of the ESR spectrum reflecting the trap-and-release process in the charge transport. The deeply trapped carriers with long trapping time, which is dominant when the charge concentration is small, give large line widths. On the other hand, shallowly trapped carriers with short trapping time become dominant for higher charge concentration regions, giving prominently narrowed ESR
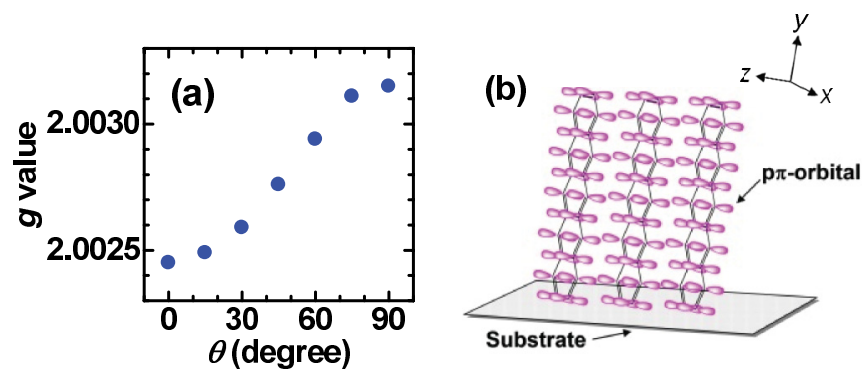

FIG. 3. (Color online) (a) Angular dependence of the $g$ value obtained with the gate voltage of $-70 \mathrm{~V}$. Here, $\theta$ denotes the angle between the magnetic field and substrate plane. (b) Schematic illustration of the average orientation of the pentacene molecules at the $\mathrm{SiO}_{2}$ interface together with the direction of $p \pi$ orbital and the definition of the principal axes of the $g$ tensor.

signals. The present result is also interpreted in the same way. The enhanced narrowing of the ESR signal in the present study for higher gate voltages provides microscopic evidence that the charge transport is affected by the trap state. The effect of charge trapping is of fundamental importance in understanding charge transport of organic thin films having the structural or energetic disorders. ${ }^{2,4,39-44}$ A remarkable consequence of this effect is a gate voltage dependence of the mobility, as has been reported for a number of OFETs. This effect is explicitly considered as a phenomenological power-law dependence of the mobility on the charge concentration in the operating devices in the next section.

The FI-ESR signal of pentacene exhibits a clear anisotropy between the data obtained with the magnetic field parallel (\|) and perpendicular $(\perp)$ to the substrate, indicating a preferential molecular orientation at the insulator interface. Figure 3(a) shows the angular dependence of the $g$ value. Here, $\theta$ denotes the angle between the magnetic field and substrate plane. The $g$ value exhibits a minimum at $\theta=0^{\circ}$ and a maximum at $\theta=90^{\circ}$, as in the cases of previous FI-ESR studies of pentacene FETs fabricated on $\mathrm{Al}_{2} \mathrm{O}_{3}$ or parylene insulators. ${ }^{31,32}$ The observed angular dependence is described well by the anisotropy of the $g$ tensor of $\pi$ electrons of pentacene, whose principal axes are shown in Fig. 3(b). Here, the $z$ axis is parallel to the $p \pi$ orbital, the $x$ axis is parallel to the $\mathrm{C}-\mathrm{H}$ bond or molecular short axis, and the $y$ axis is parallel to the molecular long axis. Recently, a DFT calculation of the $g$ values was performed for pentacene, which results in $g_{x}=2.0027, g_{y}=2.0031$, and $g_{z}=$ 2.0025 with the orthorhombic anisotropy of $g_{y}>g_{x}>g_{z} \cdot{ }^{45}$ The experimentally determined $g_{\perp}$ value of 2.0032 agrees well with the calculated $g_{y}$ value. In addition, anisotropy of $g_{\perp} \sim g_{y}=2.0031>g_{\|} \sim 1 / 2\left(g_{x}+g_{z}\right)=2.0026$ is consistent with experimental observation. These facts clearly indicate that the molecular long axis ( $y$ axis) with maximum $g$ value points almost perpendicular to the $\mathrm{SiO}_{2}$ plane, as schematically shown in Fig. 3(b).

\section{B. Charge carrier concentrations in operating devices $\left(V_{\mathrm{ds}} \neq 0\right)$}

Upon applying the drain voltage together with the gate voltage, we observe a clear decrease of the FI-ESR intensity, as shown in Fig. 4. Here, we apply a magnetic field parallel to the substrate, where the line width becomes smallest due 


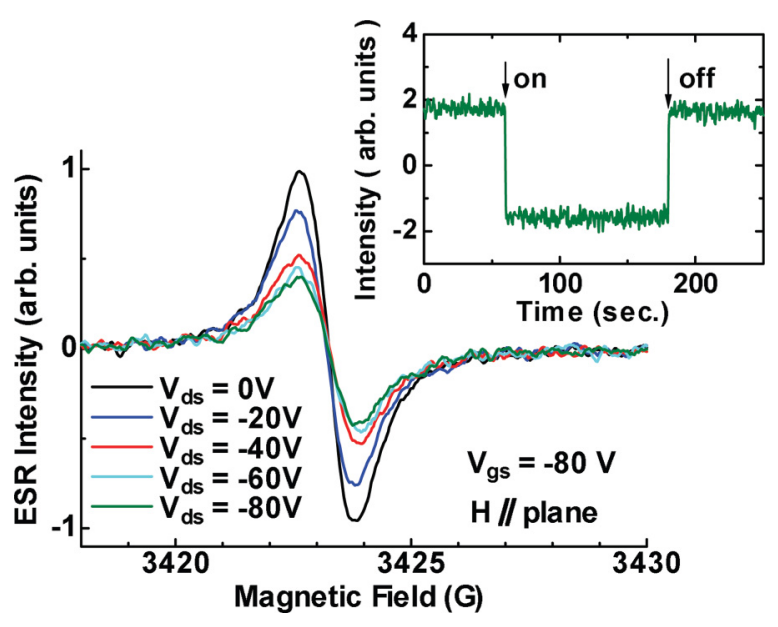

FIG. 4. (Color online) Drain voltage dependence of the firstderivative FI-ESR spectra of a pentacene FET obtained with the applied gate voltage of $-80 \mathrm{~V}$. The magnetic field is parallel to the substrate. Inset shows the time response of the FI-ESR intensity of upper peak position with respect to the application and termination of the drain voltage of $-80 \mathrm{~V}$.

to the small hyperfine interaction of the $p \pi$ orbital, ${ }^{46,47}$ to improve the $\mathrm{S} / \mathrm{N}$ ratio. The change of the signal intensity takes place reversibly within the time resolution of the present experiment $(300 \mathrm{~ms})$, as shown in the inset. This behavior clearly indicates that the total carrier number decreases by applying drain voltage, reflecting the change of charge distribution within the channel. In order to extract the spin concentration within the channel, we subtracted the spin concentration underneath the top electrodes from the total spin concentration: $C_{i}\left|V_{\mathrm{gs}}-V_{\mathrm{th}}\right| L_{s} W$ for the source electrode and $C_{i}\left|V_{\mathrm{gs}}-V_{\mathrm{th}}-V_{\mathrm{ds}}\right| L_{d} W$ for the drain electrode in the linear region. Here, $L_{s}$ and $L_{d}$ denote the length of the source and drain electrodes overlapping with the organic layer along the channel, respectively $\left[L_{s}=L_{d}=0.3 \mathrm{~mm}\right.$, as shown in Fig. 1(a)]. In the saturation region, the carrier concentration underneath the drain electrode is expected to be zero.

Figure 5(a) shows the $V_{\mathrm{ds}}$ dependence of the spin concentration within the channel normalized by the value at $V_{\mathrm{ds}}=0$ together with the corresponding output current. In the low $\left|V_{\mathrm{ds}}\right|$ region, the spin concentration exhibits a gradual decrease as the voltage increases. It reaches $72 \%$ of the initial value at $V_{\mathrm{ds}}=-60 \mathrm{~V}$, which corresponds to the pinch-off point $V_{p}\left(=V_{\mathrm{gs}}-V_{\mathrm{th}}\right)$ using $V_{\mathrm{gs}}=-80 \mathrm{~V}$ and $V_{\mathrm{th}}=-20 \mathrm{~V}$. On the other hand, it exhibits no $V_{\mathrm{ds}}$ dependence for higher voltages. Thus the spin concentration exhibits clearly different $V_{\mathrm{ds}}$ dependence between the linear $\left(\left|V_{\mathrm{ds}}\right|<\left|V_{p}\right|\right)$ and saturation $\left(\left|V_{\mathrm{ds}}\right|>\left|V_{p}\right|\right)$ regions. Then we compare this result with that expected from the change of the potential profile.

According to the GCA, local carrier concentration $Q(x)$ at a certain position $x$ within the channel is formulated by using local potential $V(x)$ and the effective gate bias $\left(V_{\mathrm{gs}}-V_{\mathrm{th}}\right)$ as $Q(x)=C_{i}\left\{V(x)-\left(V_{\mathrm{gs}}-V_{\mathrm{th}}\right)\right\}=C_{i} V_{\text {eff }}$. Here, $V_{\text {eff }}$ is the effective gate potential within the channel. The definition of $x$ is shown in Fig. 1(a). In the case of $V_{\mathrm{ds}}=0$, a uniform charge density of $C_{i}\left|V_{\mathrm{gs}}-V_{\mathrm{th}}\right|$ is induced within the channel by the applied gate bias. When the finite drain voltage is applied, a potential distribution is induced along the channel, which

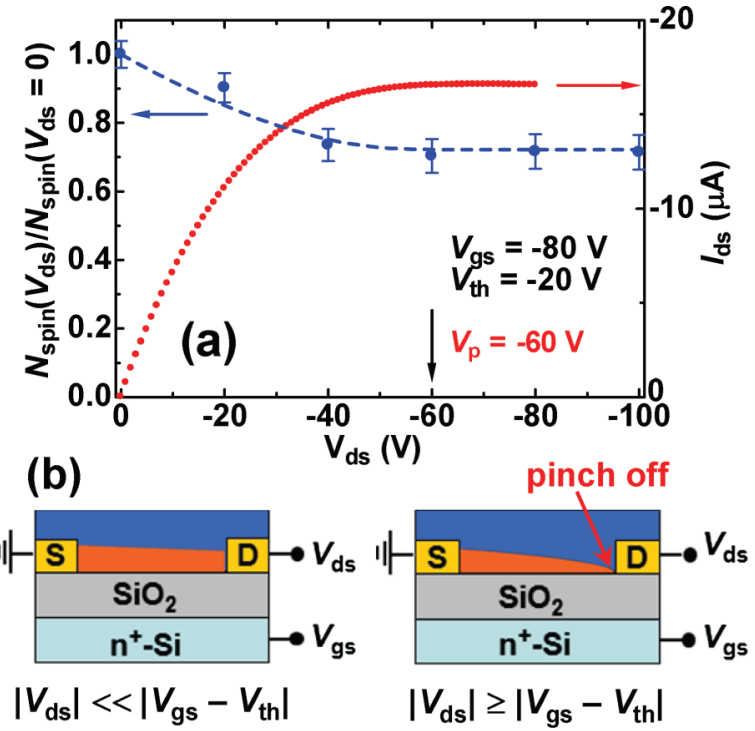

FIG. 5. (Color online) (a) Drain voltage dependence of the normalized spin concentration of a pentacene device obtained with the applied gate voltage of $-80 \mathrm{~V}$, together with the FET output current for the same device. The dashed curve represents the calculated charge concentration from the potential profile by using Eq. (7) with $\beta=2.6$. See text for details. The downward arrow shows the expected pinch-off voltage. (b) Schematic illustration of the carrier distribution, or identically potential profile, induced within the channel in the linear (left) and saturation (right) regions. Here, we show the illustration of the bottom-contact device for simplicity, which is valid because the contribution of the carriers underneath the electrodes of the top-contact device is subtracted in this study. The pinch-off region is formed around the drain electrode in the saturation region shown by the arrow.

decreases the total charge concentration within the channel. The shape of the potential distribution is nearly linear when the condition $\left|V_{\mathrm{ds}}\right| \ll\left|V_{\mathrm{gs}}-V_{\mathrm{th}}\right|$ is satisfied, as schematically shown in Fig. 5(b), because the sheet conductance is almost uniform in this condition, and the lateral electric field $\left(E_{x}=\right.$ $-d V(x) / d x$ ) along the channel is also almost uniform to fulfill the current continuity condition. In this case, the carrier concentration decreases almost linearly to the applied drain voltage as in Fig. 5(a).

On the other hand, when $\left|V_{\mathrm{ds}}\right|$ becomes comparable to $\left|V_{\mathrm{gs}}-V_{\mathrm{th}}\right|$, there are no accumulated carriers around the drain electrode, and the channel is pinched off. In the pinch-off condition, the sheet conductance decreases prominently from source to drain, and hence, the lateral electric field grows toward the drain electrode in order to satisfy the current continuity condition. In other words, the small number of carriers around the drain electrode is highly accelerated by the large lateral field so as to keep the constant current within the channel. As a result, the shape of $V(x)$ becomes nonlinear with larger gradient around the drain electrode. The shape of such nonlinear potential profile has been experimentally observed by SKPM techniques. ${ }^{18,19,23-25}$ It is also noteworthy that the growth of the lateral electric field around the drain electrode at the pinch-off point has been observed by the second harmonic generation spectroscopy in the operating pentacene transistors. ${ }^{48}$ In the saturation region of 
$\left|V_{\mathrm{ds}}\right|>\left|V_{\mathrm{gs}}-V_{\mathrm{th}}\right|$, the excess drain voltage is consumed in the narrow charge-depleted region around the drain electrode, and hence, there are no further changes of the potential shape or carrier concentration as well as the output current.

As mentioned above, a large change of the potential is induced within the channel in the pinch-off condition with boundary conditions of $\left.V_{\mathrm{eff}}\right|_{x=0}=V_{\mathrm{gs}}-V_{\mathrm{th}}$ at the source electrode and $\left.V_{\text {eff }}\right|_{x=L}=0$ at the drain electrode. If the potential drops linearly from source to drain as in the linear region, the spin concentration decreases to exactly $50 \%$ from the initial value at $\left|V_{\mathrm{ds}}\right|=\left|V_{p}\right| .{ }^{37}$ On the other hand, the observed spin concentration is clearly higher than $50 \%$, indicating that the charge distribution is superlinear with a steep drop around the drain electrode, as schematically illustrated in Fig. 5(b), consistent with the above expectations.

Since the shape of the potential profile (or carrier distribution) reflects the local conductivity through the current continuity condition as mentioned above, the observed carrier concentration in Fig. 5(a) provides microscopic information of transport properties. In the following, we calculate the change of charge carrier concentration in the operating transistors based on the GCA. Neglecting the diffusion current, the local drain current $I_{\mathrm{ds}}(x)$ is described as ${ }^{16}$

$$
I_{\mathrm{ds}}(x)=W \mu Q(x) E_{x}=-W \mu C_{i} V_{\mathrm{eff}} \frac{d V_{\mathrm{eff}}}{d x} .
$$

Here, we assume that the mobility depends on the position $x$ through the charge concentration dependence, as is expected microscopically by the enhanced motional narrowing of the FI-ESR line width for higher carrier concentrations in Fig. 2(c). In this study, we adopt a power-law dependence of the mobility on the effective gate potential ${ }^{24,49}$

$$
\mu=f_{0} V_{\mathrm{eff}}^{\beta-2},
$$

where $f_{0}$ is a prefactor. Such a power-law dependence of the mobility is expected in the case that the charge transport is dominated by the variable range hopping (VRH) process among the trap states. ${ }^{40}$ Moreover, it is also adopted empirically in the multiple trap-and-release (MTR) model. ${ }^{41,42}$ The identification of these charge transport processes is not the scope of this paper, and we use Eq. (2) as a phenomenological expression of the mobility in the present device. By integrating Eq. (1) from source $[x=0, V(x)=0]$ to drain $[x=L, V(x)=$ $\left.V_{\mathrm{ds}}\right]$, the drain current is described as $24,49,50$

$$
I_{\mathrm{ds}}=-\frac{W C_{i}}{L} \frac{f_{0}}{\beta}\left[\left(-V_{\mathrm{gs}}+V_{\mathrm{th}}\right)^{\beta}-\left(V_{\mathrm{ds}}-V_{\mathrm{gs}}+V_{\mathrm{th}}\right)^{\beta}\right] .
$$

In the saturation region, $V_{\mathrm{ds}}$ is replaced by $V_{\mathrm{gs}}-V_{\mathrm{th}}$ in Eq. (3). Then we obtain the expression

$$
I_{\mathrm{ds}}=-\frac{W C_{i}}{L} \frac{f_{0}}{\beta}\left(-V_{\mathrm{gs}}+V_{\mathrm{th}}\right)^{\beta},
$$

for the saturation region.

The conventional expressions of the drain current are obtained by putting $\beta=2$ in Eqs. (3) and (4) with the constant mobility corresponding to $f_{0}$. Equation (4) actually fits our experimental transfer curve, as shown by the solid line in the inset of Fig. 1(b). The fitting parameters are $\beta=2.6 \pm 0.1$ and $f_{0}=0.06 \mathrm{~cm}^{2} / \mathrm{V}^{(\beta-1)} \mathrm{s}$. The obtained value of $\beta$, larger than
2 , gives evidence for the effective gate voltage dependence of the mobility.

Then we calculate the potential profile and the total carrier number within the channel. By using Eq. (3), the local current at the position $x$ becomes

$$
I_{\mathrm{ds}}(x)=-\frac{W C_{i}}{x} \frac{f_{0}}{\beta}\left\{\left(-V_{\mathrm{gs}}+V_{\mathrm{th}}\right)^{\beta}-\left[V(x)-V_{\mathrm{gs}}+V_{\mathrm{th}}\right]^{\beta}\right\} .
$$

From the current continuity condition of $I_{\mathrm{ds}}(x)=I_{\mathrm{ds}}$, the local potential is solved as ${ }^{24}$

$$
\begin{aligned}
V(x)= & V_{\mathrm{gs}}-V_{\mathrm{th}}+\left\{\left(-V_{\mathrm{gs}}+V_{\mathrm{th}}\right)^{\beta}-\frac{x}{L}\left[\left(-V_{\mathrm{gs}}+V_{\mathrm{th}}\right)^{\beta}\right.\right. \\
& \left.\left.-\left(V_{\mathrm{ds}}-V_{\mathrm{gs}}+V_{\mathrm{th}}\right)^{\beta}\right]\right\}^{\frac{1}{\beta}} .
\end{aligned}
$$

This equation actually fits the experimental potential profile as determined from SKPM technique. ${ }^{23-25}$ The total carrier number is then obtained by integrating the local carrier concentration within the channel as

$$
\begin{aligned}
& e N_{\text {charge }} \\
& \quad=W \int_{0}^{L} C_{i} V_{\mathrm{eff}} d x \\
& =L W C_{i} \frac{\beta}{\beta+1} \frac{\left(-V_{\mathrm{gs}}+V_{\mathrm{th}}\right)^{\beta+1}-\left(V_{\mathrm{ds}}-V_{\mathrm{gs}}+V_{\mathrm{th}}\right)^{\beta+1}}{\left(-V_{\mathrm{gs}}+V_{\mathrm{th}}\right)^{\beta}-\left(V_{\mathrm{ds}}-V_{\mathrm{gs}}+V_{\mathrm{th}}\right)^{\beta}} .
\end{aligned}
$$

When $V_{\mathrm{ds}}$ approaches 0 , Eq. (7) converges to $-L W C_{i}\left(V_{\mathrm{gs}}-\right.$ $\left.V_{\text {th }}\right)$, as expected. The dashed curve in Fig. 5(a) shows $V_{\mathrm{ds}}$ dependence of the carrier number calculated by Eq. (7) in the linear region of $\left|V_{\mathrm{ds}}\right| \leqslant 60 \mathrm{~V}$, which is normalized by the value at $V_{\mathrm{ds}}=0$. We adopted the parameter values of $\beta=2.6$ and $V_{\mathrm{th}}=-20 \mathrm{~V}$, which are determined from the transfer characteristics and the $V_{\mathrm{gs}}$ dependence of the spin concentration, respectively. Note that the dashed curve in Fig. 5(a) is extended to the saturation region with the same value at $V_{\mathrm{ds}}=V_{p}(=-60 \mathrm{~V})$ because no further change of carrier number is expected in this region. The agreement between the experimental and calculated values is satisfactory. Here, the ratio of the spin concentration at the pinch-off point and at $V_{\mathrm{ds}}=0$ acts as a simple measure of the $\beta$ value; it becomes $\beta /(\beta+1)$ as readily expected from Eq. (7). In the case of $\beta=2$ for the uniform mobility, the spin concentration becomes $67 \%$. In the present pentacene device, the experimentally determined value of $72 \%$ agrees well with the case of $\beta=2.6$. It is also noteworthy that the pinch-off voltage is reasonably determined from the shape of the $V_{\mathrm{ds}}$ dependence of the spin concentration, as shown by the downward arrow in Fig. 5(a). It shifted consistently by changing the applied gate voltage following the relation $V_{p}=V_{\mathrm{gs}}-V_{\mathrm{th}}$.

In order to further confirm the validity of the discussion above, we also applied the FI-ESR technique to the operating P3HT transistor. Figure 6 shows the $V_{\mathrm{ds}}$ dependence of the FI-ESR signal obtained by applying negative gate voltage of $V_{\mathrm{gs}}=-10 \mathrm{~V}$. The magnetic field is perpendicular to the substrate. At $V_{\mathrm{ds}}=-0 \mathrm{~V}$, we observe a clear 


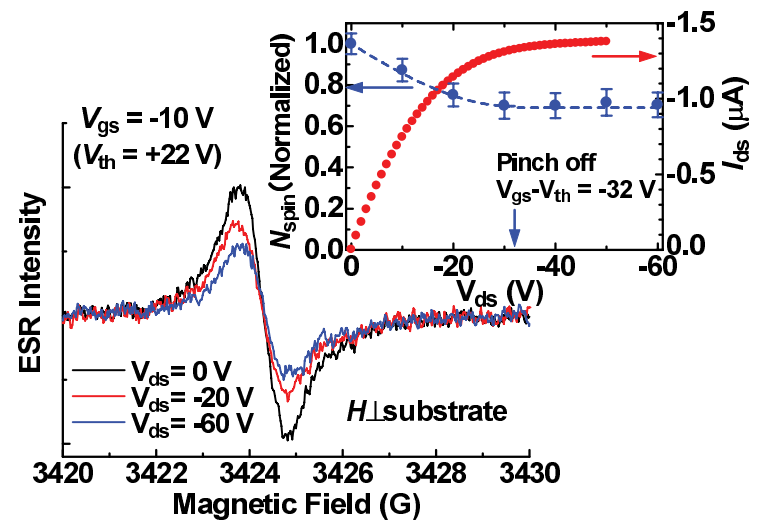

FIG. 6. (Color online) Drain voltage dependence of the firstderivative FI-ESR spectra of a P3HT FET obtained with the applied gate voltage of $-10 \mathrm{~V}$. The magnetic field is perpendicular to the substrate. Inset shows the $V_{\mathrm{ds}}$ dependence of the spin concentration together with the FET output current for the same device. The dashed curve represents the calculated charge concentration from the potential profile by using Eq. (7) with $\beta=2.3$. The downward arrow shows the expected pinch-off voltage.

FI-ESR signal of positive polarons on the chain around $g=$ 2.003. The observed $g$ value corresponds well with that expected for the molecular short axis, as determined in the uniaxially oriented P3HT ultrathin transistors ${ }^{29}$ indicating that the P3HT chain takes edge-on orientation at the insulator interface to form the lamellar structure. By applying drain voltages, a clear decrease of the FI-ESR intensity is observed as in the case of the pentacene device. Here, the applied gate voltage is sufficiently small not to induce the spinless states, which has been observed in the high gate voltage region in previous FI-ESR studies due to the formation of spinless bipolarons (or polaron pairs). ${ }^{26-28,37}$ Thus, $N_{\text {spin }}$ agrees with the charge concentration.

The inset of Fig. 6 shows the $V_{\mathrm{ds}}$ dependence of $N_{\text {spin }}$ normalized by the value at $V_{\mathrm{ds}}=0 \mathrm{~V}$ together with the FET output current. Here, $N_{\text {spin }}$ decreases to $70 \%$ of the initial value in the saturation region. The transfer characteristics of the same device was fitted well by Eq. (3) in this device, and the extracted parameters were $f_{0}=7 \times 10^{-3} \mathrm{~cm}^{2} / \mathrm{V}^{(\beta-1)} \mathrm{s}, \beta=$ 2.3 , and $V_{\text {th }}=22 \mathrm{~V}$. By using these parameters, we calculated the drain voltage dependence of the carrier number by Eq. (7) as shown by the dashed curve. The agreement between the experimental and calculated results is quite satisfactory. In addition, the pinch-off voltage, shown by the downward arrow, takes different values as that determined for the pentacene device in Fig. 5(a) due to the different $V_{\mathrm{gs}}$ and $V_{\text {th }}$ values.

It is also noteworthy that similar $V_{\mathrm{ds}}$ dependence of the spin concentration has recently been observed in the operating rubrene single-crystal transistor with bottom-contact geometry. ${ }^{38}$ Note that the bottom-contact device has no carriers underneath the electrodes, and all the FI-ESR signals come from the channel carriers. The carrier concentration decreases to $\sim 60 \%$ of the initial value in the saturation region, higher than $50 \%$, indicating that the potential profile is also nonlinear, consistent with this study. On the other hand, the carrier concentration ratio is clearly smaller than the present thin film devices of $\sim 70 \%$. This may indicate that the gate voltage dependence of the mobility is negligible in the rubrene device, although the carrier concentration ratio is even smaller than the expected value in the case of $\beta=2$. This result is supported by the transfer characteristics showing a linear $I_{\mathrm{ds}}{ }^{1 / 2}-V_{\mathrm{gs}}$ dependence, ${ }^{36}$ as well as the narrow and $V_{\mathrm{gs}}$-independent FI-ESR line width in the rubrene devices. ${ }^{38}$ These facts may indicate that the rubrene single-crystal transistor, fabricated by the lamination technique, produces few deep trap states, resulting in the high charge carrier mobilities.

The above results provide direct evidence that the device characteristics are intrinsically dominated by the change of the carrier concentration within the channel, which reflects the change of potential profile, both in the thin film and single crystalline transistors. This indicates the validity of GCA in understanding the operation principle of these organic transistors. The FI-ESR spectroscopy is thus a useful method to obtain device parameters, such as carrier concentration, threshold voltage or pinch-off voltage, gate-voltage dependence of the mobility, as well as the dynamical feature of the carriers. On the other hand, further details of the charge transport, such as the microscopic origin of the trap state as well as the identification of the transport model, are still unclear in this study. In this context, application of this method at low temperatures may be an interesting subject to understand these properties, which is left open for further studies.

\section{CONCLUSION}

In this paper, we demonstrated the change of charge carrier concentration in the operating OFETs of pentacene and P3HT through the direct determination of spin concentration by using the FI-ESR technique. The FI-ESR signals of positive polarons were clearly observed by applying negative gate biases in both devices. Upon applying drain voltage, the carrier concentration decreased in the linear region, reaching $\sim 70 \%$ of the initial value at the pinch-off point, and stayed constant in the saturation region. The change of the carrier concentration reflects the change of the charge distribution, or identically the potential profile based on the gradual channel approximation, within the channel. Here, $V_{\mathrm{ds}}$ dependence of the carrier concentration was reproduced well by the potential calculation considering the power-law dependence of the mobility on the effective gate potential as $\mu \propto V_{\text {eff }}^{\beta-2}$, as was also confirmed from the transfer characteristic of the device. The spin concentration decreases to $\beta /(\beta+1)$ of the initial value at the pinch-off voltage, providing a simple measure to estimate $\beta$ as well as the pinch-off voltage. Obtained values of $\beta$ were $\beta=2.6$ for a pentacene device and $\beta=2.3$ for a P3HT device, consistent with those obtained by the transfer characteristics. The gate voltage dependence of the mobility, which reflects a carrier trapping at the device interface, was further confirmed microscopically from the enhanced motional narrowing of the FI-ESR signal for higher gate voltages.

\section{ACKNOWLEDGMENT}

This work has been partially supported by Grant-in-Aid for Scientific Research (Grants No. 22340080 and No. 24654087) from the JSPS and Tatematsu Foundation. 
${ }^{1} \mathrm{Z}$. Bao and J. Locklin, Organic Field-Effect Transistors (CRC Press, Boca Raton, 2007).

${ }^{2}$ H. Sirringhaus, Adv. Mater. 17, 2411 (2005).

${ }^{3}$ M. E. Gershenson, V. Podzorov, and A. F. Morpurgo, Rev. Mod. Phys. 78, 973 (2006).

${ }^{4}$ H. Klauk, Chem. Soc. Rev. 39, 2643 (2010).

${ }^{5}$ M. Kitamura and Y. Arakawa, J. Phys.: Condens. Matter 20, 184011 (2008).

${ }^{6}$ J. E. Anthony, J. S. Brooks, D. L. Eaton, and S. R. Parkin, J. Am. Chem. Soc. 123, 9482 (2001).

${ }^{7}$ J. E. Anthony, Chem. Rev. 106, 5029 (2006).

${ }^{8}$ H. Ebata, T. Izawa, E. Miyazaki, K. Takimiya, M. Ikeda, H. Kuwabara, and T. Yui, J. Am. Chem. Soc. 129, 15732 (2007).

${ }^{9}$ K. Takimiya, S. Shinamura, I. Osaka, and E. Miyazaki, Adv. Mater. 23, 4347 (2011).

${ }^{10}$ I. McCulloch, M. Heeney, C. Bailey, K. Genevicius, I. MacDonald, M. Shkunov, D. Sparrowe, S. Tierney, R. Wagner, W. Zhang, M. L. Chabinye, R. J. Kline, M. D. McGehee, and M. F. Toney, Nat. Mater. 5, 328 (2006).

${ }^{11}$ T. Umeda, D. Kumaki, and S. Tokito, J. Appl. Phys. 105, 124516 (2009).

${ }^{12}$ H. N. Tsao, D. M. Cho, I. Park, M. R. Hansen, A. Mavrinskiy, D. Y. Yoon, R. Graf, W. Pisula, H. W. Spiess, and K. Müllen, J. Am. Chem. Soc. 133, 2605 (2011).

${ }^{13}$ H. Bronstein, Z. Chen, R. S. Ashraf, W. Zhang, J. Du, J. R. Durrant, P. S. Tuladhar, K. Song, S. E. Watkins, Y. Geerts, M. M. Wienk, R. A. J. Janssen, T. Anthopoulos, H. Sirringhaus, M. Heeney, and I. McCulloch, J. Am. Chem. Soc. 133, 3272 (2011).

${ }^{14}$ S. Kobayashi, T. Nishikawa, T. Takenobu, S. Mori, T. Shimoda, T. Mitani, H. Shimotani, N. Yoshimoto, S. Ogawa, and Y. Iwasa, Nat. Mater. 3, 317 (2004).

${ }^{15}$ L. Miozzo, A. Abderrahim, and G. Horowitz, J. Mater. Chem. 20, 2513 (2010).

${ }^{16}$ S. M. Sze, Physics of Semiconductor Devices, 2nd ed. (John Wiley \& Sons, New York, 1981).

${ }^{17}$ C. R. Newman, C. D. Frisbie, D. A. Silva Filho, J. L. Brédas, P. C. Ewbank, and K. R. Mann, Chem. Mater. 16, 4436 (2004).

${ }^{18}$ L. Bürgi, H. Sirringhaus, and R. H. Friend, Appl. Phys. Lett. 80, 2913 (2002).

${ }^{19}$ L. Bürgi, T. J. Richards, R. H. Friend, and H. Sirringhaus, J. Appl. Phys. 94, 6129 (2003).

${ }^{20}$ K. P. Puntambekar, P. V. Pesanento, and C. D. Frisbie, Appl. Phys. Lett. 83, 5539 (2003).

${ }^{21}$ M. Nakamura, N. Goto, N. Ohashi, M. Sakai, and K. Kudo, Appl. Phys. Lett. 86, 122112 (2005).

${ }^{22}$ T. Hallam, M. J. Lee, N. Zhao, I. Nandhakumar, M. Kemerink, M. Heeney, I. McCulloch, and H. Sirringhaus, Phys. Rev. Lett. 103, 256803 (2009).

${ }^{23}$ L. Bürgi, T. J. Richards, M. Chiesa, R. H. Friend, and H. Sirringhaus, Synth. Met. 146, 297 (2004).

${ }^{24}$ E. C. P. Smits, S. G. J. Mathijssen, M. Cölle, A. J. G. Mank, P. A. Bobbert, P. W. M. Blom, B. de Boer, and D. M. de Leeuw, Phys. Rev. B 76, 125202 (2007).
${ }^{25}$ M. Kemerink, T. Hallam, M. J. Lee, N. Zhao, M. Caironi, and H. Sirringhaus, Phys. Rev. B 80, 115325 (2009).

${ }^{26}$ K. Marumoto, Y. Muramatsu, Y. Nagano, T. Iwata, S. Ukai, H. Ito, S. Kuroda, Y. Shimoi, and S. Abe, J. Phys. Soc. Jpn. 74, 3066 (2005).

${ }^{27}$ S. Watanabe, K. Ito, H. Tanaka, H. Ito, K. Marumoto, and S. Kuroda, Jpn. J. Appl. Phys. 46, L792 (2007).

${ }^{28} \mathrm{~S}$. Kuroda, S. Watanabe, K. Ito, H. Tanaka, H. Ito, and K. Marumoto, Appl. Magn. Reson. 36, 357 (2009).

${ }^{29}$ S. Watanabe, H. Tanaka, S. Kuroda, A. Toda, S. Nagano, T. Seki, A. Kimoto, and J. Abe, Appl. Phys. Lett. 96, 173302 (2010).

${ }^{30}$ S. Watanabe, H. Tanaka, H. Ito, S. Kuroda, T. Mori, K. Marumoto, and Y. Shimoi, Org. Electron. 12, 716 (2011).

${ }^{31}$ K. Marumoto, S. I. Kuroda, T. Takenobu, and Y. Iwasa, Phys. Rev. Lett. 97, 256603 (2006).

${ }^{32}$ H. Matsui, T. Hasegawa, Y. Tokura, M. Hiraoka, and T. Yamada, Phys. Rev. Lett. 100, 126601 (2008).

${ }^{33}$ H. Matsui, A. S. Mishchenko, and T. Hasegawa, Phys. Rev. Lett. 104, 056602 (2010).

${ }^{34}$ H. Tanaka, M. Kozuka, S. I. Watanabe, H. Ito, Y. Shimoi, K. Takimiya, and S. I. Kuroda, Phys. Rev. B 84, 081306(R) (2011).

${ }^{35}$ H. Matsui, D. Kumaki, E. Takahashi, K. Takimiya, S. Tokito, and T. Hasegawa, Phys. Rev. B 85, 035308 (2012).

${ }^{36}$ K. Marumoto, N. Arai, H. Goto, M. Kijima, K. Murakami, Y. Tominari, J. Takeya, Y. Shimoi, H. Tanaka, S. I. Kuroda, T. Kaji, T. Nishikawa, T. Takenobu, and Y. Iwasa, Phys. Rev. B 83, 075302 (2011).

${ }^{37}$ H. Tanaka, S. Watanabe, H. Ito, K. Marumoto, and S. Kuroda, Appl. Phys. Lett. 94, 103308 (2009).

${ }^{38}$ M. Tsuji, N. Arai, K. Marumoto, J. Takeya, Y. Shimoi, H. Tanaka, S. Kuroda, T. Takenobu, and Y. Iwasa, Appl. Phys. Express 4, 085702 (2011).

${ }^{39}$ R. Coehoorn, W. F. Pasveer, P. A. Bobbert, and M. A. J. Michels, Phys. Rev. B 72, 155206 (2005).

${ }^{40}$ M. C. J. M. Vissenberg and M. Matters, Phys. Rev. B 57, 12964 (1998).

${ }^{41}$ G. Horowitz, M. E. Hajlaoui, and R. Hajlaoui, J. Appl. Phys. 87, 4456 (2000).

${ }^{42}$ R. Bourguiga, M. Mahdouani, S. Mansouri, and G. Horowitz, Eur. Phys. J. Appl. Phys. 39, 7 (2007).

${ }^{43}$ A. Salleo, T. W. Chen, A. R. Völkel, Y. Wu, P. Liu, B. S. Ong, and R. A. Street, Phys. Rev. B 70, 115311 (2004).

${ }^{44}$ R. A. Street, J. E. Northrup, and A. Salleo, Phys. Rev. B 71, 165202 (2005).

${ }^{45}$ Y. Shimoi, K. Marumoto, and S. Kuroda, Mol. Cryst. Liq. Cryst. 566, 33 (2012).

${ }^{46}$ J. R. Morton, Chem. Rev. 64, 453 (1964).

${ }^{47}$ S. Kuroda, Int. J. Mod. Phys. B 9, 221 (1995).

${ }^{48}$ M. Weis, T. Manaka, and M. Iwamoto, J. Appl. Phys. 105, 024505 (2009).

${ }^{49}$ E. C. P. Smits, T. D. Anthopoulos, S. Setayesh, E. van Veenendaal, R. Coehoorn, P. W. M. Blom, B. de Boer, and D. M. de Leeuw, Phys. Rev. B 73, 205316 (2006).

${ }^{50}$ O. Simonetti, L. Giraudet, T. Maurel, J.-L. Nicolas, and A. Belhhir, Org. Electron. 11, 1381 (2010). 